\title{
APTT-LA Screen to Confirm Percent Difference
}

National Cancer Institute

\section{Source}

National Cancer Institute. APTT-LA Screen to Confirm Percent Difference. NCI

Thesaurus. Code C161372.

A measurement to confirm the presence of Lupus anticoagulants, calculated as [(Screen aPTT - Confirm aPTT)/Screen aPTT]x100. 